\title{
A survey of national and multi-national registries and cohort studies in juvenile idiopathic arthritis: challenges and opportunities
}

Timothy Beukelman ${ }^{1,19^{*}}$, Janneke Anink ${ }^{2}$, Lillemor Berntson ${ }^{3}$, Ciaran Duffy ${ }^{4}$, Justine A. Ellis ${ }^{5}$, Mia Glerup ${ }^{6}$, Jaime Guzman ${ }^{7}$, Gerd Horneff ${ }^{8}$, Lianne Kearsley-Fleet ${ }^{9}$, Ariane Klein ${ }^{8}$, Jens Klotsche ${ }^{10}$, Bo Magnusson ${ }^{11}$, Kirsten Minden ${ }^{10}$, Jane E. Munro ${ }^{12}$, Martina Niewerth ${ }^{10}$, Ellen Nordal ${ }^{13}$, Nicolino Ruperto ${ }^{14}$, Maria Jose Santos ${ }^{15}$, Laura E. Schanberg ${ }^{16}$, Wendy Thomson ${ }^{9}$, Lisette van Suijlekom-Smit ${ }^{2}$, Nico Wulffraat ${ }^{17}$ and Kimme Hyrich ${ }^{18}$

\begin{abstract}
Background: To characterize the existing national and multi-national registries and cohort studies in juvenile idiopathic arthritis (JIA) and identify differences as well as areas of potential future collaboration.

Methods: We surveyed investigators from North America, Europe, and Australia about existing JIA cohort studies and registries. We excluded cross-sectional studies. We captured information about study design, duration, location, inclusion criteria, data elements and collection methods.

Results: We received survey results from 18 studies, including 11 national and 7 multi-national studies representing 37 countries in total. Study designs included inception cohorts, prevalent disease cohorts, and new treatment cohorts (several of which contribute to pharmacosurveillance activities). Despite numerous differences, the data elements collected across the studies was quite similar, with most studies collecting at least 5 of the 6 American College of Rheumatology core set variables and the data needed to calculate the 3-variable clinical juvenile disease activity score. Most studies were collecting medication initiation and discontinuation dates and were attempting to capture serious adverse events.

Conclusion: There is a wide-range of large, ongoing JIA registries and cohort studies around the world. Our survey results indicate significant potential for future collaborative work using data from different studies and both combined and comparative analyses.
\end{abstract}

Keywords: Juvenile idiopathic arthritis, Registry, Observational study, Pharmacosurveillance, Pediatric rheumatology

\section{Background}

Juvenile idiopathic arthritis (JIA), a heterogeneous collection of inflammatory arthritides, is the most common rheumatic condition of childhood [1]. Despite this, our understanding of the long-term outcomes for many children with this condition, in terms of disease status,

\footnotetext{
* Correspondence: tbeukelman@peds.uab.edu

'University of Alabama at Birmingham, Birmingham, USA

${ }^{19}$ Division of Pediatric Rheumatology, The University of Alabama at Birmingham, 1600 7th Avenue South, CPP 210, Birmingham, AL 35233-1711, USA

Full list of author information is available at the end of the article
}

functional limitation, need for long-term immunosuppression as well as the development of comorbidities, remains relatively limited. Similarly, we have an incomplete understanding of the potential adverse effects of new therapeutic agents, especially effects that are rare or have a long latency period.

The epidemiological study of JIA is challenging. Compared to the $1 \%$ prevalence of rheumatoid arthritis (RA) in adults, JIA is rare. The approximate worldwide yearly incidence is 8 per 100,000 children and most prevalence estimates range from 15 to 150 per 100,000 depending on geographic region and 
study methodology [2]. In contrast to the categorization of the arthritis counterparts in adulthood, JIA encompasses a wide range of inflammatory arthritides, from selflimited oligoarthritis to severe, persistent polyarthritis, and it also includes psoriatic arthritis, spondyloarthritis, and systemic arthritis (an autoinflammatory condition). Results from long-term follow-up studies of JIA may not be broadly generalizable; they may exclude children with milder forms of disease who are discharged from rheumatology care, and they may include too few children to adequately study the less common disease phenotypes. The long-term study of childhood-onset diseases is complicated when patients transition to the adult healthcare setting, making the capture of health outcome data challenging. However, patients and parents identify long-term outcomes, such as physical ability in adulthood, successful schooling and employment, and the consequences of long-term exposure to immunosuppression in childhood, as some of their key priorities for research $[3,4]$.

With the advent of biological therapies and their increasing use, reliable outcome data in JIA has become even more crucial. Little is known about the background rates of serious outcomes, such as infections and malignancies, making it difficult to interpret the safety of new medications. Extrapolation from studies of these drugs in adults with RA has limited accuracy because most children with JIA do not have RA (only approximately $5 \%$ have rheumatoid factor positive polyarthritis) and children have far fewer serious comorbid conditions compared to adults. Exposure to immune modulating drugs in developing immune systems may also result in different longer-term adverse health outcomes compared to more mature immune systems.

Given the rarity of JIA and the now frequent use of potentially high risk treatments in the absence of robust, large, long-term safety studies, the capture of data in longitudinal cohorts is essential. Appropriately combining data from various cohorts enables detection of rare adverse events and more accurate assessment of disease outcomes. Comparing data from different countries may provide information about genotypic variations in disease and geographic variation in environmental risk factors. It may also allow for comparisons of treatment differences that result largely from local availability of therapeutic agents rather than differences in disease characteristics.

The purpose of this study was to survey the international landscape of prospective longitudinal registries and cohort studies in JIA to assess the breadth and depth of data being collected. This information will inform future international collaborative work in JIA, including data harmonization for future studies.

\section{Methods}

A list of existing JIA registries and cohort studies was compiled through known contacts of the authors, ongoing collaborations, and a review of the recent literature. This included studies conducted in the United States of America (USA), Canada, United Kingdom (UK), Germany, the Netherlands, Sweden, Norway, Portugal, and Australia, as well as some multi-national studies. A survey developed in Microsoft Excel (2010) was emailed to the principal investigators of each registry or cohort. The survey also asked for additional registries or cohorts known to the respondent. Surveys were completed between October and December 2014. Owing to delays in manuscript preparation and in an effort to provide the most recent information possible, all respondents were given the opportunity to revise their responses in March 2016.

The survey captured details about each registry or cohort study, including study design, study duration, location, inclusion criteria and number of participants, and data elements and collection method. Cross-sectional studies, defined as those with less than 12 months of planned follow-up per participant, were excluded.

\section{Results}

In total, 20 investigators were contacted, and the replies described 20 studies. Two identified studies were not included (CLARITY from Australia [5] and EPOCA from PRINTO [6]) due to cross-sectional design. The remaining 18 studies contain more than 60,000 patients with JIA (Table 1). These studies include 11 national and 7 multi-national cohorts (covering more than 37 countries in total), with data spanning from 1993 until 2016 onwards. These included 5 JIA inception cohorts (recruiting exclusively from disease onset), 6 prevalent disease cohorts (without strict requirements about disease duration or treatment), and 7 treatment cohorts (restricted to children starting certain anti-rheumatic therapies, such as biologics). Data from treatment cohorts were often part of pharmacosurveillance regulatory requirements. A majority of studies had been established within the past 15 years. Despite the long duration of some studies, the number of total subjects recruited to each study remained relatively low, with few national studies exceeding 1500 children.

Most studies are still recruiting and continuing to followup children previously recruited. The planned length of follow-up varied across studies and was generally dependent on the amount or nature of funding available (data not shown). A majority of studies were based in a single country or a small number of countries within a geographic area. One noted exception is the large multinational Pharmacovigilance in Juvenile Idiopathic Arthritis Patients (Pharmachild) collaboration,which was initiated with a European Union grant from the Framework 
Beukelman et al. Pediatric Rheumatology (2017) 15:31

Page 3 of 9

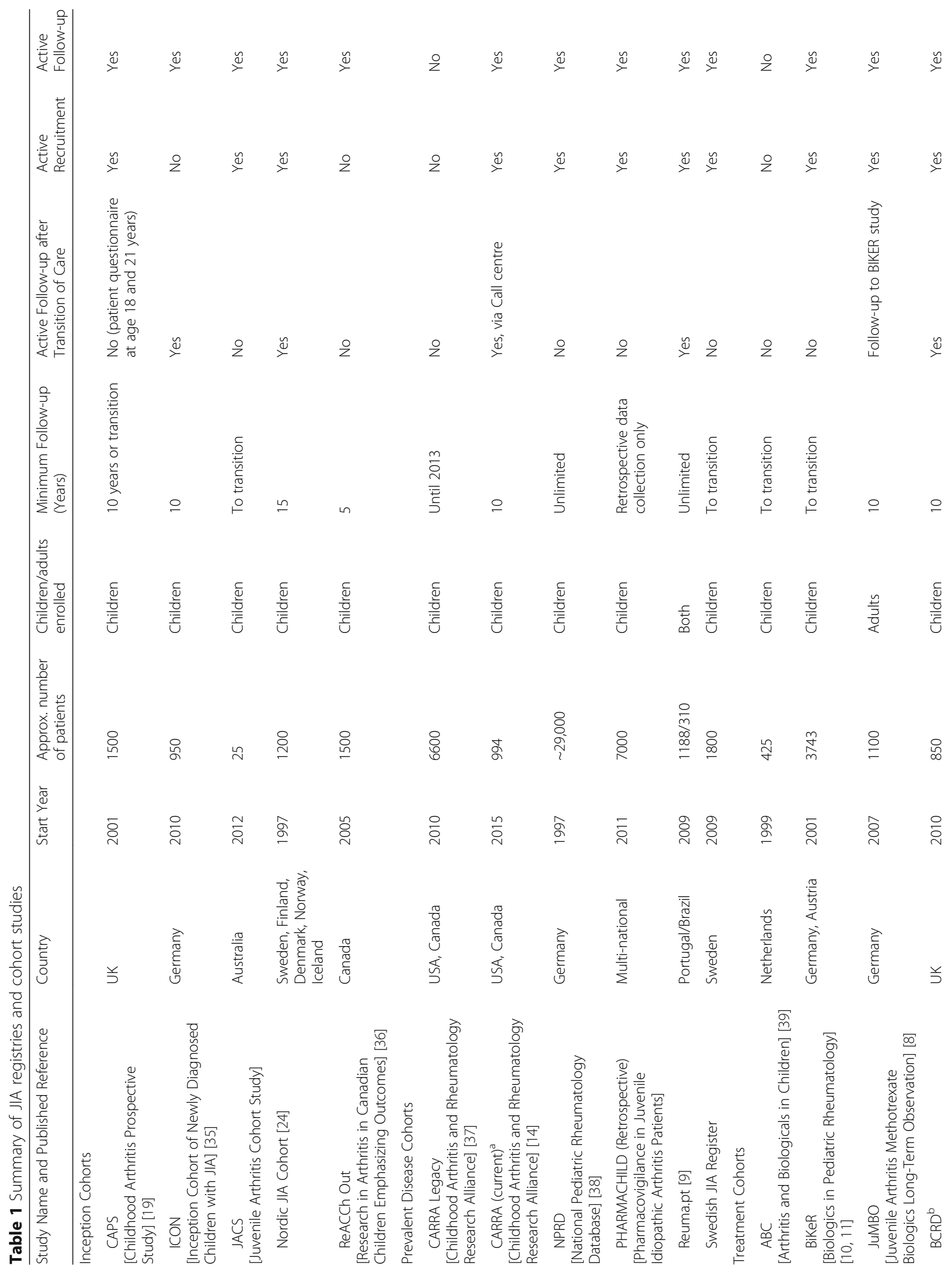




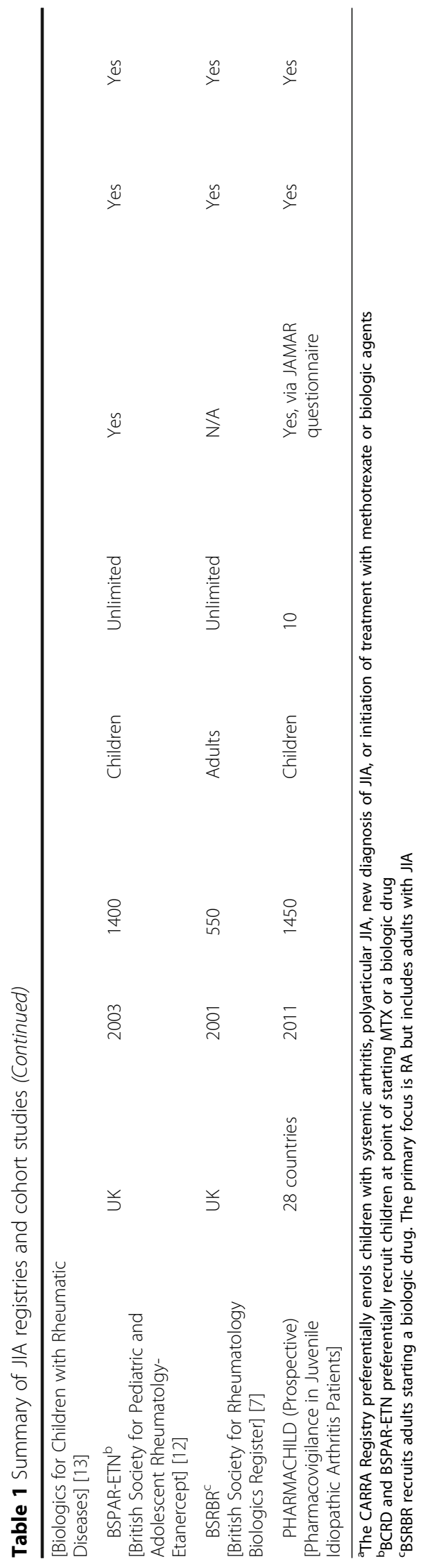


Beukelman et al. Pediatric Rheumatology (2017) 15:31

Page 5 of 9

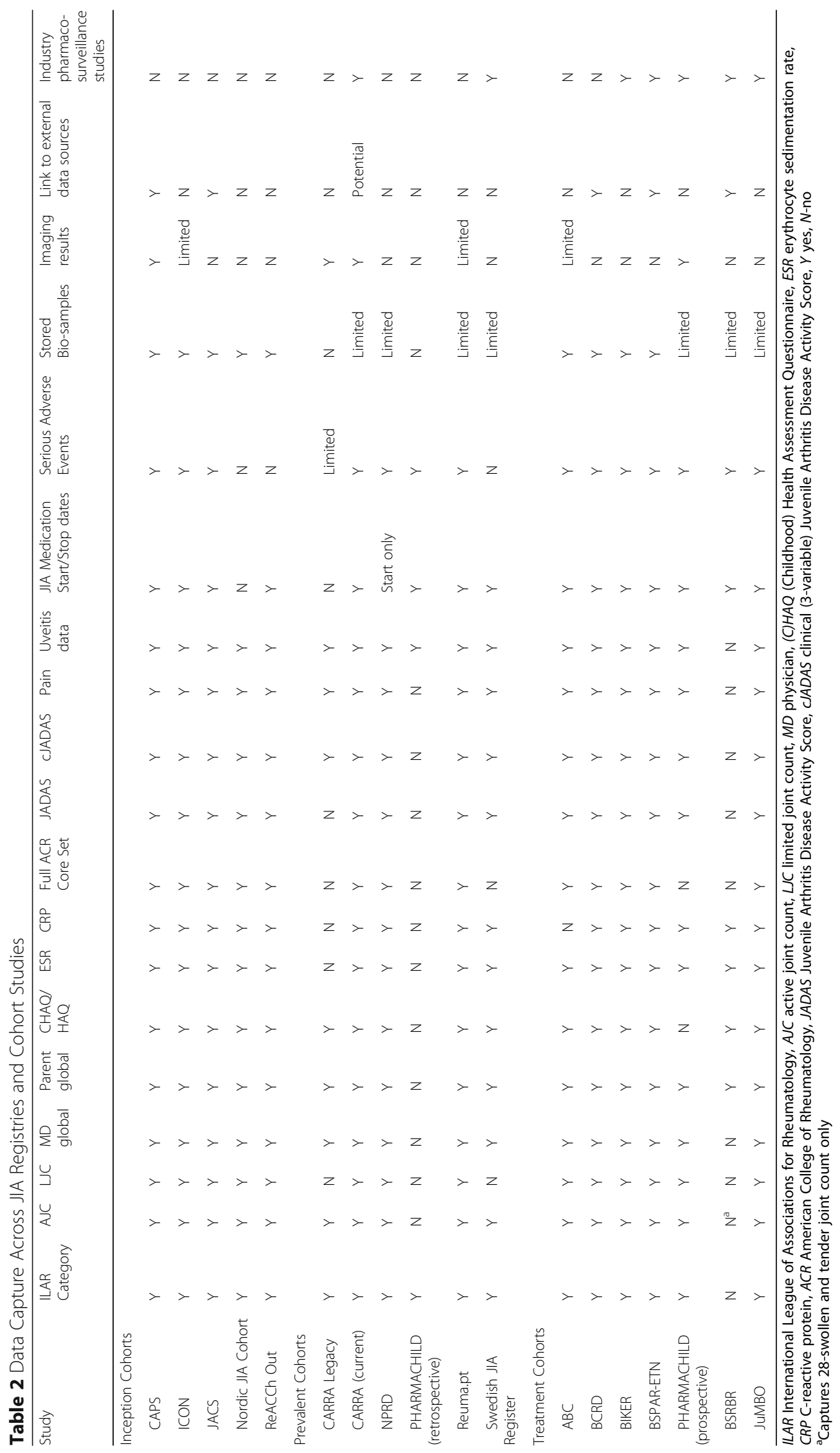


Programme 7, managed by the Pediatric Rheumatology International Trials Organization (PRINTO), and has recruited children from 28 countries.

The vast majority of studies recruited children, although 2 studies (The British Society for Rheumatology Biologics Register (BSRBR) [7] and The German Juvenile Arthritis - Methotrexate/Biologics Long-term Observation Study (JUMBO) [8], specifically recruit adult patients with JIA receiving biologic therapies. Most studies stop follow-up after children leave paediatric care; however, there are several exceptions. The Portuguese register, Reuma.pt, is built into routine care and continues to capture data from patients into adulthood [9]. JUMBO is a follow-on study that links adults who were followed in the German BiKeR registry $[10,11]$ as children. Similarly, the UK BSPAR [12] and BCRD registers [13] continue to capture outcome data about young adults from their adult care providers. The CARRA Registry follows young adults after transition of care via a structured survey conducted by a telephone call center [14]. Pharmachild has the option for adult rheumatology centers to submit data and plans to collect data directly from young adults after transition of care by use of the JAMAR questionnaire [15].

Despite differences in geographic location, time since study inception, and study designs, the data elements collected across studies appears to be quite similar (Table 2). All studies but one capture the International League of Associations for Rheumatology (ILAR) category of JIA [1]. More than two-thirds of studies capture the full American College of Rheumatology JIA core outcome set (count of joints with active arthritis, count of joints with limited range of motion, physician global assessment of overall disease activity, patient/parent global assessment of overall well-being, functional ability (Childhood Health Assessment Questionnaire (CHAQ) score), inflammatory markers) [16]. Nearly all of the remaining studies capture 5 of the ACR core outcome variables. Fifteen studies (83\%) collect the data needed to calculate the 10 or 71-joint Juvenile Arthritis Disease Activity Score (JADAS-10 or JADAS-71) [17], and 16 can calculate the 3-variable clinical JADAS (cJADAS) which omits inflammatory markers [18]. All studies enrolling children captured data on the presence or absence of uveitis. Most studies (83\%) captured medication start and stop dates. Fourteen studies $(78 \%)$ captured the occurrence of serious adverse events (e.g., requiring hospitalisation), but only $28 \%$ had the ability to capture these data additionally through external data sources (the majority based in the UK). Seven registries were working with industry to perform pharmacosurveillance studies and included monitoring of data and adjudication of adverse event reports.
Biosample collection was performed in most of the registries. Nine registries had relatively systematic collection, and 7 had more limited collection on a subset of patients. Serum, plasma, and whole blood/DNA were the most commonly collected samples. Synovial fluid was collected less frequently.

\section{Discussion}

This is the first report to bring together information about the numerous existing JIA registries and longitudinal cohort studies, highlighting a wealth of JIA outcome data being collected around the world. Many important questions surround the etiology, pathogenesis, optimal management, and long-term outcomes of JIA. No single registry can answer all questions. Registries must have a clearly defined purpose that determines the process of data collection and the specific data items collected.

Three primary patient enrolment strategies are being used: diagnosis inception cohorts, prevalence or convenience cohorts, and treatment initiation cohorts. Although all studies aim to assess disease outcomes over time, inception cohorts better identify predictors of short and long-term outcomes (e.g. social, clinical, psychological, laboratory and genetic) [19], whereas treatment initiation cohorts are superior to evaluate treatment effectiveness and safety. Of course, with sufficient patient enrolment and procedures to collect data at times when medications are newly started, treatment cohort studies can be performed within a subset of patients from any registry.

Whilst one study started collecting data as early as 1993, most initiated recruitment following the introduction of biologic therapies for JIA around 2000. This was likely a direct result of increased monitoring and safety concerns among investigators, sponsors, and patients with JIA. The relatively recent increase of JIA cohort studies coincided with a movement in the late 1990's to classify children with chronic arthritis into homogenous groups to facilitate research using the ILAR classification [1], and this is reflected by all paediatric studies classifying children according to this system. Most studies continue to actively recruit and follow previously recruited patients.

Unfortunately, many studies cease to follow patients after they transition into adult care. The transition of care period presents many challenges. Patients not only change clinicians and often their place of residence, but also may change medical systems and third-party payers. The most relevant outcome measures may also change, such as a change from active and limited joint counts in paediatric clinics to counts of tender and swollen joints in adult clinics, or change from JADAS to RA disease activity measures (e.g., DAS28). 
With the majority of JIA studies initiating in the 2000 's only preliminary results on adult-aged patients are currently available. Two studies specifically assess effectiveness and safety of biologics in adult patients with JIA. The German JuMBO study has published outcome data of 346 children (median age 21 years) who had received the biologic etanercept in childhood [8]. The UK BSRBR study has published data on outcomes of 225 adults with JIA who started a biological therapy in childhood [7]. In previous studies, outcomes of adults with JIA have been assessed cross-sectionally [20-22], retrospectively [23], or by identifying adult patients diagnosed as children from medical records [24-27]. These studies reported approximately 40 to $60 \%$ of children with JIA continue to have some level of active disease in adulthood [22, 24, 25, 27-29]. Given the relative rarity of JIA and that not all children continue to have active disease in adulthood, it will be important for registries to share their experiences to develop robust methods to track children through adulthood. Because few patients enrolled in the current studies have reached adulthood, this may be an opportunity now to align the data to be collected in the future across studies.

The studies included in our survey do not reflect the entirety of long-term outcomes studies in JIA. Several recent studies have used administrative data to investigate the risk of rare outcomes in JIA, such as malignancy or serious infection [30-32]. Administrative data sources provide readily available data and often very large sample sizes, but provide scant clinical data compared to clinical cohorts. Accordingly, administrative data are typically most useful for studies of adverse effects of medications, but are less useful for studies of medication effectiveness or long-term outcomes in JIA.

Although there were many differences among the studies, there was significant overlap in the data being collected. This may allow for the determination of a minimal data set that all existing and future prospective observational studies could collect, enabling nearly identical analysis plans to be conducted across all data sources.

Our survey was able to summarize the extent of data items captured, but did not collect estimates of the amount of missing data for each variable. Missing data are a common problem in observational research due to clinic non-attendance by patients, variable clinical need for investigation (e.g. inflammatory marker and other blood testing), and failure to report adverse events in busy outpatient clinics, among others. This can result in biases that require careful consideration of how to handle missing data and missing patients. This issue is compounded further in JIA due to the heterogeneity of disease, with many children who are well being discharged from secondary care and therefore no longer contributing to longer term outcome studies. Also, our survey did not enquire in detail about the timing of data capture, particularly in relation to disease onset or start of therapies.

In order to understand the risk of rare outcomes, large sample sizes and long term follow up are needed. Combining data from multiple studies increases statistical power, but must be done carefully. It is reassuring that there is significant commonality on the data items captured across studies, which may permit the creation of a common data model to pool data. However, differing methods of outcome ascertainment (e.g., assessing adverse events by patient report, physician report, or through an external data source) may produce important differences in results. It will also be important to ensure that no patients are double-counted if they have been simultaneously enrolled in more than one registry effort; this is likely currently best addressed at the individual clinical site level, but assignment of a universal identification number allowing patients to contribute to various different registries over time (e.g., in response to changes in age or geographic location) would have potential additional benefits.

This survey did not account for the differences in health care systems, clinical expertise in JIA, or funding for arthritis medications around the world. Because patients are enrolled from pediatric rheumatology centers, differences in health care systems may influence enrolment into JIA registries through physician referral bias. Differential access to biologics likely results in substantial differences in disease duration and severity at the time of starting treatment. Given the differences in patient enrolment and data collection techniques, as well as geographical differences in treatment approaches, genetic background of the patients, and varying incidences of comorbid conditions and endemic pathogens (such as tuberculosis), simple pooling of data may not be the most appropriate approach to evaluate drug safety or effectiveness. In addition, there may be restrictions on the sharing of data, depending on the ethical approval and patient consent obtained in each study. Overcoming these hurdles to facilitate collaborative research in JIA will be challenging and will require an international effort involving multiple stakeholders. Within the JIA research community, initial steps have been taken to begin to address these obstacles [33].

Sophisticated methods for combining data should be explored, such as nested case-control studies or metaanalyses of individual register data analysis. These approaches were adopted by the EULAR Registers and Observational Drug Studies (RODS) Working Group to explore the risk of malignant melanoma associated with TNFi therapies in patients with RA across Europe [34]. Methodological approaches that consider the individual 
data source will likely provide more accurate results and new insights about confounders and effect modifiers of outcome. Despite the significant resources required, these analyses would be a unique opportunity to answer critical questions about JIA and should be undertaken.

\section{Conclusions}

This study identified a wide range of ongoing JIA cohort studies and registries around the world. Many of the challenges in the long-term study of childhood-onset diseases were highlighted. Nevertheless, the results indicate significant potential for future collaborative work using both combined and comparative analyses of data from different studies. New approaches to maximise data capture beyond childhood will be crucial to answer important questions about long-term outcomes.

\begin{abstract}
Abbreviations
ABC: Arthritis and biologicals in children; ACR: American college of rheumatology; AJC: Active joint count; BCRD: Biologics for children with rheumatic diseases; BiKeR: Biologics in pediatric rheumatology; BSPARETN: British society for pediatric and adolescent rheumatolgy-etanercept; BSRBRC: British society for rheumatology biologics register; CAPS: Childhood arthritis prospective study; CARRA: Childhood arthritis and rheumatology research alliance; CHAQ: Childhood health assessment questionnaire; CRP: C-reactive protein; ESR: Erythrocyte sedimentation rate; HAQ: Health assessment questionnaire; ICON: Inception cohort of newly diagnosed children with jia; ILAR: International league of associations for rheumatology; JACS: Juvenile arthritis cohort study; JADAS: Juvenile arthritis disease activity score; JAMAR: Juvenile arthritis multidimensional assessment report; JIA: Juvenile idiopathic arthritis; JuMBO: Juvenile arthritis methotrexate biologics long-term observation; LJC: Limited joint count; MD: Physician; N: No; NPRD: National pediatric rheumatology database; Pharmachild: Pharmacovigilance in juvenile idiopathic arthritis patients; PRINTO: Pediatric rheumatology international trials organization; RA: Rheumatoid arthritis; ReACCh Out: Research in arthritis in canadian children emphasizing outcomes; RODS: Registers and observational drug studies; UK: United Kingdom; USA: United States of America; Y: Yes
\end{abstract}

\section{Acknowledgements}

The authors thank all children with JIA and their families who have contributed to these studies.

ICON: coordinator Angela Zink; consortium partners Dirk Foell and Arnd Heiligenhaus; funded by a research grant of the Federal Ministry of Education and Research (FKZ 01ER0812); made possible by the support and participation of more than 300 German paediatric and adult rheumatologists whom we thank.

NPRD: funded by the German child arthritis foundation; made possible by the support and participation of more than 300 German paediatric and adult rheumatologists whom we thank.

JuMBO: Co-PI Angela Zink; funded by an unconditional grant from Pfizer and Abbvie; made possible by the support and participation of more than 300 German paediatric and adult rheumatologists whom we thank. CAPS: funded by Arthritis Research UK (Grant \# 20542) BCRD: funded by Arthritis Research UK (Grant \# 20747)

BSRBR: funded by British Society for Paediatric and Adolescent Rheumatology and the British Society for Rheumatology BSPAR-ETN: funded by British Society for Paediatric and Adolescent Rheumatology and the British Society for Rheumatology CARRA Legacy Registry: funded by United States National Institutes of Health/National Institute of Arthritis and Musculoskeletal and Skin Diseases (1RC2AR058934), Friends of CARRA, Arthritis Foundation

CARRA Registry: funded by Arthritis Foundation, Novartis, Roche; Executive Committee members Laura Schanberg, Yukiko Kimura, Timothy Beukelman, Jason Jones, and Helen Bristow
JACS: Justine Ellis was supported by an Australian Research Council Future Fellowship, FT120100253

Pharmachild: funded by European Union Framework Programme 7 grant (260353); Chiara Pallotti (PRINTO coordination center)

BiKeR: made possible by the collaboration of numerous German and Austrian pediatric rheumatologists, patients and their parents and by non-restricted grants from Abbvie, Chugai, Novartis, Pfizer, and Roche. Swedish JIA Register: Board of the Registry

Reuma.pt: made possible by the collaboration of pediatric and adult rheumatologists, rheumatology nurses, patients, and by unrestricted grants from AbbVie, Celegene, Hospira, MSD, Pfizer, Roche, and UCB.

Nordic JIA Study: conducted by the Nordic Study Group of Pediatric Rheumatology (NoSPeR)

\section{Funding}

This work was supported in part by the Arthritis Research UK Centre for Epidemiology (Grant \#20380). The funding body had no role in the design of the study or the collection, analysis, or interpretation of the data, or the writing of the manuscript.

\section{Availability of data and materials}

Data sharing is not applicable to this article as no datasets were generated or analysed during the current study.

\section{Authors' contributions}

The study was conceived by TB, LES, and KH. All authors contributed to the acquisition and interpretation of data. The manuscript was drafted by TB, $\mathrm{LKF}$, and $\mathrm{KH}$. All authors revised the manuscript critically and gave approval of the final version.

\section{Competing interests}

The authors declare that they have no competing interests.

\section{Consent for publication}

Not applicable

\section{Ethics approval and consent to participate}

Not applicable

\section{Publisher's Note}

Springer Nature remains neutral with regard to jurisdictional claims in published maps and institutional affiliations.

\section{Author details \\ ${ }^{1}$ University of Alabama at Birmingham, Birmingham, USA. ${ }^{2}$ Erasmus Medical Center, Rotterdam, The Netherlands. ${ }^{3}$ Uppsala University Hospital, Uppsala, Sweden. ${ }^{4}$ Children's Hospital of Eastern Ontario, Ottawa, Canada. ${ }^{5}$ Murdoch Children's Research Institute, Genes, Environment \& Complex Disease, Parkville, Australia. ${ }^{6}$ Aarhus University Hospital, Aarhus, Denmark. ${ }^{7}$ University of British Columbia, Vancouver, Canada. ${ }^{8}$ Asklepios Klinik Sankt Augsutin, Sankt Augustin, Germany. ${ }^{9}$ University of Manchester, Manchester, UK. ${ }^{10}$ German Rheumatism Research Center, Berlin, Germany. ${ }^{11}$ Karolinska University Hospital, Stockholm, Sweden. ${ }^{12}$ Royal Children's Hospital, Melbourne, Australia. ${ }^{13}$ University Hospital of North Norway, Tromso, Norway. ${ }^{14}$ Istituto Giannina Gaslini, Genoa, Italy. ${ }^{15}$ Hospital Garcia de Orta, Almada, Portugal. ${ }^{16}$ Duke University, Durham, USA. ${ }^{17}$ University Medical Center Utrecht, Utrecht, The Netherlands. ${ }^{18}$ University of Manchester and Central Manchester Foundation Trust, Manchester, UK. ${ }^{19}$ Division of Pediatric Rheumatology, The University of Alabama at Birmingham, 1600 7th Avenue South, CPP 210, Birmingham, AL 35233-1711, USA.}

Received: 25 January 2017 Accepted: 7 April 2017

Published online: 19 April 2017

\section{References}

1. Petty RE, Southwood TR, Manners P, Baum J, Glass DN, Goldenberg J, et al. International league of associations for rheumatology classification of juvenile idiopathic arthritis: second revision, Edmonton, 2001. J Rheumatol. 2004:31:390-2.

2. Thierry S, Fautrel B, Lemelle I, Guillemin F. Prevalence and incidence of juvenile idiopathic arthritis: a systematic review. Joint Bone Spine. 2014;81:112-7. 
3. Guzman J, Gomez-Ramirez O, Jurencak R, Shiff NJ, Berard RA, Duffy CM, et al. What matters most for patients, parents, and clinicians in the course of juvenile idiopathic arthritis? a qualitative study. J Rheumatol. 2014;41: 2260-9.

4. van Pelt P, Wulffraat NM. Transition of care in rheumatology: managing the rheumatic patient from childhood to adulthood. In: Harrison A, editor. Insights and perspectives in rheumatology: InTech. 2012. p. 255-74.

5. Ellis JA, Ponsonby AL, Pezic A, Chavez RA, Allen RC, Akikusa JD, et al. CLARITY - ChiLdhood arthritis risk factor identification sTudY. Pediatr Rheumatol Online J. 2012;10:37.

6. Consolaro A, Ruperto N, Filocamo G, Lanni S, Bracciolini G, Garrone M, et al. Seeking insights into the EPidemiology, treatment and outcome of childhood arthritis through a multinational collaborative effort: introduction of the EPOCA study. Pediatr Rheumatol Online J. 2012;10:39.

7. McErlane F, Foster HE, Davies R, Lunt M, Watson KD, Symmons DP, et al. Biologic treatment response among adults with juvenile idiopathic arthritis: results from the British society for rheumatology biologics register. Rheumatology (Oxford). 2013;52:1905-13.

8. Minden K, Niewerth M, Zink A, Seipelt E, Foeldvari I, Girschick H, et al. Long-term outcome of patients with JIA treated with etanercept, results of the biologic register JuMBO. Rheumatology (Oxford). 2012;51:1407-15.

9. Canhao H, Faustino A, Martins F, Fonseca JE. Rheumatic diseases Portuguese register board coordination PSoR. Reuma.pt - the rheumatic diseases Portuguese register. Acta Reumatol Port. 2011;36:45-56.

10. Horneff G, De Bock F, Foeldvari I, Girschick HJ, Michels H, Moebius D, et al. Safety and efficacy of combination of etanercept and methotrexate compared to treatment with etanercept only in patients with juvenile idiopathic arthritis (JIA): preliminary data from the German JIA registry. Ann Rheum Dis. 2009;68:519-25.

11. Schmeling H, Minden K, Foeldvari I, Ganser G, Hospach T, Horneff G. Efficacy and safety of adalimumab as the first and second biologic agent in juvenile idiopathic arthritis: the German biologics JIA registry. Arthritis Rheumatol. 2014;66:2580-9.

12. Davies R, Southwood TR, Kearsley-Fleet L, Lunt M, Hyrich KL, British Society for $P$, et al. Medically significant infections Are increased in patients with juvenile idiopathic arthritis treated with etanercept: results from the British society for paediatric and adolescent rheumatology etanercept cohort study. Arthritis Rheumatol. 2015;67:2487-94.

13. Kearsley-Fleet $L$, Davies R, Baildam E, Beresford MW, Foster HE, Southwood TR, et al. Factors associated with choice of biologic among children with juvenile idiopathic arthritis: results from two UK paediatric biologic registers. Rheumatology (Oxford). 2016:55:1556-65.

14. Beukelman T, Kimura Y, llowite NI, Mieszkalski K, Natter MD, Burrell G, et al. The new Childhood Arthritis and Rheumatology Research Alliance (CARRA) Registry: design, rationale, and characteristics of patients enrolled in the first 12 months. (in press).

15. Filocamo G, Consolaro A, Schiappapietra B, Dalpra S, Lattanzi B, MagniManzoni S, et al. A new approach to clinical care of juvenile idiopathic arthritis: the juvenile arthritis multidimensional assessment report. J Rheumatol. 2011;38:938-53.

16. Giannini EH, Ruperto N, Ravelli A, Lovell DJ, Felson DT, Martini A. Preliminary definition of improvement in juvenile arthritis. Arthritis Rheum. 1997;40:1202-9.

17. Consolaro A, Ruperto N, Bazso A, Pistorio A, Magni-Manzoni S, Filocamo G, et al. Development and validation of a composite disease activity score for juvenile idiopathic arthritis. Arthritis Rheum. 2009;61:658-66.

18. McErlane F, Beresford MW, Baildam EM, Chieng SE, Davidson JE, Foster HE, et al. Validity of a three-variable juvenile arthritis disease activity score in children with new-onset juvenile idiopathic arthritis. Ann Rheum Dis. 2013;72:1983-8.

19. Adib N, Hyrich K, Thornton J, Lunt M, Davidson J, Gardner-Medwin J, et al. Association between duration of symptoms and severity of disease at first presentation to paediatric rheumatology: results from the childhood arthritis prospective study. Rheumatology (Oxford). 2008;47:991-5.

20. Packham JC, Hall MA. Long-term follow-up of 246 adults with juvenile idiopathic arthritis: functional outcome. Rheumatology (Oxford). 2002;41:1428-35.

21. Bruinooge LN, El Moussaoui R, Taal E, Dijkmans BA, Van Soesbergen RM. Outcome in adults with juvenile idiopathic arthritis. Comparison of the DUTCH-AIMS2 between JIA and RA. Scand J Rheumatol. 2003;32:89-94.

22. Foster HE, Marshall N, Myers A, Dunkley P, Griffiths ID. Outcome in adults with juvenile idiopathic arthritis: a quality of life study. Arthritis Rheum. 2003:48:767-75
23. Peterson LS, Mason T, Nelson AM, O'Fallon WM, Gabriel SE. Psychosocial outcomes and health status of adults who have had juvenile rheumatoid arthritis: a controlled, population-based study. Arthritis Rheum. 1997;40:2235-40.

24. Nordal E, Zak M, Aalto K, Berntson L, Fasth A, Herlin T, et al. Ongoing disease activity and changing categories in a long-term nordic cohort study of juvenile idiopathic arthritis. Arthritis Rheum. 2011;63:2809-18.

25. Zak M, Pedersen FK. Juvenile chronic arthritis into adulthood: a long-term follow-up study. Rheumatology (Oxford). 2000;39:198-204.

26. French AR, Mason T, Nelson AM, O'Fallon WM, Gabriel SE. Increased mortality in adults with a history of juvenile rheumatoid arthritis: a population-based study. Arthritis Rheum. 2001:44:523-7.

27. Arkela-Kautiainen M, Haapasaari J, Kautiainen $\mathrm{H}$, Leppanen L, Vilkkumaa I,

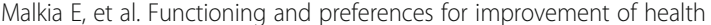
among patients with juvenile idiopathic arthritis in early adulthood using the WHO ICF model. J Rheumatol. 2006:33:1369-76.

28. Fantini F, Gerloni V, Gattinara M, Cimaz R, Arnoldi C, Lupi E. Remission in juvenile chronic arthritis: a cohort study of 683 consecutive cases with a mean 10 year followup. J Rheumatol. 2003;30:579-84.

29. Selvaag AM, Aulie HA, Lilleby $V$, Flato B. Disease progression into adulthood and predictors of long-term active disease in juvenile idiopathic arthritis. Ann Rheum Dis. 2016;75:190-5.

30. Beukelman T, Xie F, Chen L, Baddley JW, Delzell E, Grijalva CG, et al. Rates of hospitalized bacterial infection associated with juvenile idiopathic arthritis and its treatment. Arthritis Rheum. 2012;64:2773-80.

31. Beukelman T, Haynes K, Curtis JR, Xie F, Chen L, Bemrich-Stolz CJ, et al. Rates of malignancy associated with juvenile idiopathic arthritis and its treatment. Arthritis Rheum. 2012;64:1263-71.

32. Nordstrom BL, Mines D, Gu Y, Mercaldi C, Aquino P, Harrison MJ. Risk of malignancy in children with juvenile idiopathic arthritis not treated with biologic agents. Arthritis Care Res (Hoboken). 2012;64:1357-64.

33. Robinson E, Albani S, Beresford MW, de Jager W, de Roock S, Duong T, et al. Towards stratified medicine in juvenile idiopathic arthritis [abstract]. 2016 Pediatric Rheumatology European Society (PReS) Congress.

34. Mercer LK, Askling J, Raaschou P, Dixon WG, Dreyer L, Hetland ML, et al. Risk of invasive melanoma in patients with rheumatoid arthritis treated with biologics: results from a collaborative project of 11 European biologic registers. Ann Rheum Dis. 2017;76(2):386-91.

35. Sengler C, Klotsche J, Niewerth M, Liedmann I, Foll D, Heiligenhaus A, et al. The majority of newly diagnosed patients with juvenile idiopathic arthritis reach an inactive disease state within the first year of specialised care: data from a German inception cohort. RMD open. 2015;1:e000074.

36. Oen K, Tucker L, Huber AM, Miettunen P, Scuccimarri R, Campillo S, et al. Predictors of early inactive disease in a juvenile idiopathic arthritis cohort: results of a Canadian multicenter, prospective inception cohort study. Arthritis Rheum. 2009;61:1077-86.

37. Beukelman T, Ringold S, Davis TE, DeWitt EM, Pelajo CF, Weiss PF, et al. Disease-modifying antirheumatic drug use in the treatment of juvenile idiopathic arthritis: a cross-sectional analysis of the CARRA Registry. J Rheumatol. 2012;39:1867-74.

38. Minden K, Niewerth M, Listing J, Zink A. German study group of pediatric R. Health care provision in pediatric rheumatology in Germany-national rheumatologic database. J Rheumatol. 2002;29:622-8.

39. Prince FH, Twilt M, ten Cate R, van Rossum MA, Armbrust W, Hoppenreijs $E P$, et al. Long-term follow-up on effectiveness and safety of etanercept in juvenile idiopathic arthritis: the Dutch national register. Ann Rheum Dis. 2009;68:635-41. 